\title{
The Role of Telomeres in Cancer Development and Progression, and the Double Edge Sword Effect with Tamoxifen
}

\section{Nezami MA ${ }^{1 *}$, Hager $\mathbf{S}^{2}$ and Garner $\mathbf{J}^{1}$}

${ }^{1}$ Research Cancer Institute of America, Pacific Medical Center of Hope, Fresno, CA 93720, USA

${ }^{2}$ Ccare, Fresno, CA, USA

\section{Keywords: Tamoxifen; Cancer prevention; Telomeres}

\section{Introduction}

The current practice regarding the use of estrogen receptor antagonists in breast cancer, (such as Tamoxifen), has opened doors for investigators to research these drugs for their use in the prevention of breast cancer. Although this approach is suggested by the literature to be an effective strategy, currently there are no established guidelines applicable to the use of anti-estrogen receptor drugs or prevention in the clinical setting $[1,2]$.

We review the literature related to Tamoxifen therapy and recommend expansion of the application of low dose Tamoxifen for patients with positive estrogen receptor cancers to preventive oncology. These applications include patients at risk for hormonally responsive cancers, and expand beyond that to individuals who may not be even at risk as defined by current risk stratification standards, such as strong family history of breast cancer. In this article we also review an opposing negative effect of Tamoxifen in patients with active cancer, and discuss why we believe it should be "avoided" in the treatment of solid tumors (except breast cancer), although it may be a meaningful tool in prevention of cancer of all types.

\section{Background}

The double edge sword of Tamoxifen (favorable in prevention and unfavorable in therapy), is based on our novel understanding of telomeres and their role in carcinogenesis and cancer progression [3-6]. Further studies are warranted in application of low dose Tamoxifen to prevent all solid tumors in unaffected individuals, in a safe and efficacious manner. The theory of how Tamoxifen interacts with telomeres also provides a basic understanding of possible unfavorable mechanisms involved with Tamoxifen's application and its resistance in treated tumors.

\section{Telomeres and Tamoxifen}

Tamoxifen is shown in the literature to be a telomerase activator [7]. The activation of telomeres is an important step in carcinogenesis as it is secondary to what is known as a "telomere crisis" $[3,8]$. As we age, the length of the telomeres shortens. Continuation of this process over time is important, as it causes the cell DNA to be exposed to genomic instability. This instability at a critical length of telomeres promotes apoptosis, by activating the P53 and other onco-suppressor genes in normal circumstances.

However, if this instability continues beyond a certain point, and/ or if the P53 system is impaired, or if the cell is exposed to a telomerase activator (such as Tamoxifen), it can cause DNA damage and mutations in both oncogene promotors as well as oncogene suppressors, which can promote the cancer development at the first stages (carcinogenesis). It is therefore postulated that the telomere crisis happens long before the DNA mutations required for carcinogenesis. The unstable DNA shares the same alterations identified in cancer. Finally, activation of telomerase can save the cell from apoptosis, and death. This activation is uniform in almost all cancer cells, with high proliferative index [4] shown by both tumor tissue biopsy as well as liquid biopsies. Although it is suggested that telomerase activity is not required for malignant transformation, and it merely an index for highly proliferative cells, it is commonly used as a marker for detection and monitoring the cancer cells, both in tissue biopsies as well as circulating tumor cells or CTCs (Telomerase is an indicator for presence of tumor epithelial cells in the blood) $[9,10]$.

Studies have shown that by using tamoxifen in a cellular culture, the ability of the cells to activate telomerase increases, and as the result the cell will continue to stay young, with prolonged resistance to crisis [7]. This can prevent the cell from an increased amount of genomic instability and further carcinogenesis. However, the same mechanism can explain the cancerous cell's ability to survive longer in the presence of Tamoxifen, as a telomerase activator [7]. The cancer cells exposed to 4-hydroxy Tamoxifen in culture are able to survive longer and infiltrate distant organs in mice studies, explaining the increased invasion. The critical question remains whether the application of Tamoxifen as early as possible in individuals not known to have cancer may prevent the telomere crisis, and if so, it can prevent carcinogenesis long before it is clinically diagnosed.

Tamoxifen therapy also may have unintended effects on gene expression. This theory is presented in the following two cases studies, both patients with breast cancer who had positive circulating tumor DNA for Neurofibromatosis-I gene post tamoxifen therapy.

\section{Case Studies}

\section{Breast cancer with positive NF-1}

70 year old female with history of left breast ductal carcinoma diagnosed in 2001 status post lumpectomy and hormonal therapy. Hormonal therapy consisted of Femara for 3 years and Arimidex for 4 years. She was also treated with radiation, and was status-post recurrence of the tumor in same side documented in September 2013, through core biopsy and referral for surgery (mastectomy), and chemotherapies. The extent of disease was documented to involve the skin and required neoadjuvant chemotherapies. Her left breast had a $5 \mathrm{~cm}$ mass. She was seen and evaluated for complimentary medicine, and immediately started on IV epigenetic therapies after initial labs. Her initial labs showed extensively elevated angiogenic markers, including Interleukin 8 (both in serum and plasma). Her Interleukin 8 was 103.3

*Corresponding author: Nezami MA, Research Cancer Institute of America Pacific Medical Center of Hope, Fresno, CA 93720, USA, Tel: (559) 475 4300; E-mail: amnezami@yahoo.com

Received: February 23, 2016; Accepted March 18, 2016; Published March 21 2016

Citation: Nezami MA, Hager S, Garner J (2016) The Role of Telomeres in Cancer Development and Progression, and the Double Edge Sword Effect with Tamoxifen. Biol Med (Aligarh) 8: 296. doi: 10.4172/0974-8369.1000296

Copyright: (c) 2016 Nezami MA, et al. This is an open-access article distributed under the terms of the Creative Commons Attribution License, which permits unrestricted use, distribution, and reproduction in any medium, provided the original author and source are credited. 
Citation: Nezami MA, Hager S, Garner J (2016) The Role of Telomeres in Cancer Development and Progression, and the Double Edge Sword Effect with Tamoxifen. Biol Med (Aligarh) 8: 296. doi: 10.4172/0974-8369.1000296

Page 2 of 4

in plasma (normal less than 34) and 136 in serum (normal less than 66), measured on 6/3/14. Treatment was given every day in first two weeks, five days a week, and twice a week after. She was started on Tamoxifen at a dose of $5 \mathrm{mg}$ daily. Her labs were repeated on 6/17/14 and 8/29/14. She reported improved QOL while receiving therapies and no side effects experienced. Her labs showed marked reduction in both markers. Her IL-8 in serum dropped down to 89 and in her plasma down to 16.8. Further this dropped down to normal range. Her Interleukin 8 normalized both in serum as well as plasma (50 in serum and 27 in plasma), on 10/8/2014.

She subsequently underwent surgery on $12 / 14$, and currently is in remission. She received Firmagon injection, as well as Cytoxan (metronomic dose) along with our therapy, and her VEGF dropped down to less than 9 , on $5 / 28 / 15$. Her circulatory tumor DNA (ctDNA) was studied through Guardant $360 \mathrm{Lab}$, on 6/25/15, which showed positive NF-1, and APC, likely due to the exposure to anti-estrogen therapy. Following further treatments with MTET (Multi-targeted Epigenetic therapy), her Guardant 360 was repeated and showed no ctDNA (Figure 1).

This anti-angiogenetic response to the therapies in a novel approach needs to be investigated further in clinical trials to reduce the risk of recurrence and improve response to conventional treatments.

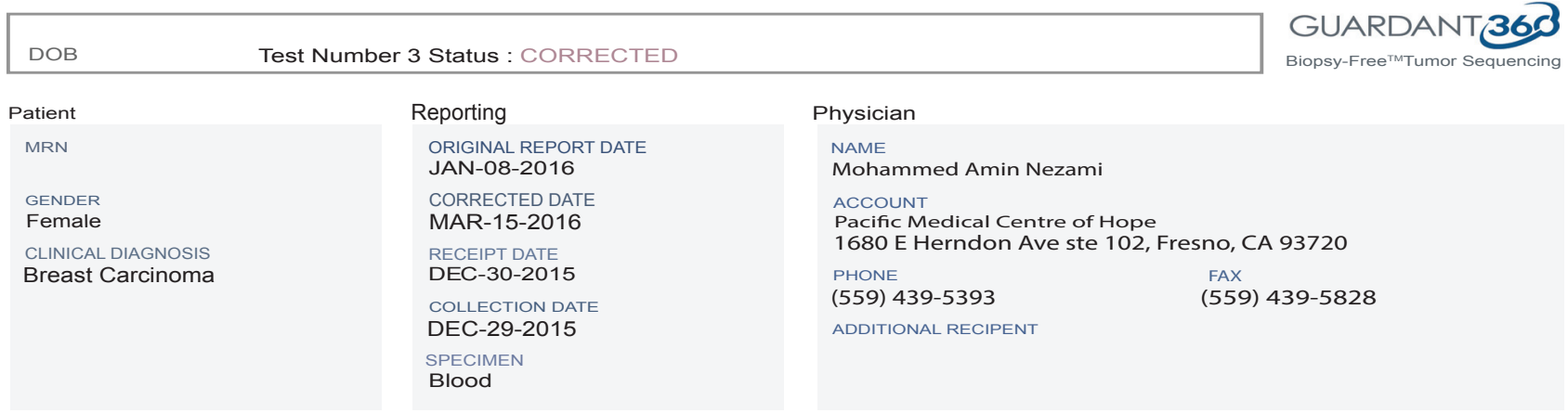

Guardant360 Tumor Response Map

The Guardant 360 Tumor Response Map illustrates the relative changes of observed cfDNA at different samples submission time points. The "somatic Alteration Burden" value below refers to the maximum \% cfDNA detected at each time point. Amplifications are not plotted and only the first and last four test dates are plotted. please see the physician portal for the Tumor Response Mao with all test dates.

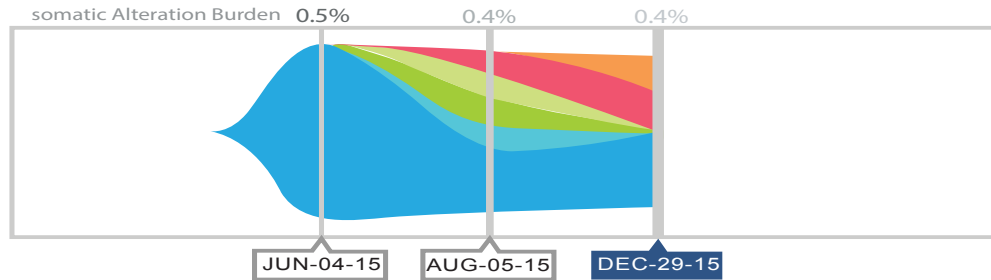

Summary of Alterations \& Associated Treatment Options

The percantage, or allele frequency, of altered cell-free DNA (\% cfDNA) circulating in blood is related to the unique tumor biology of this patient. Factors that may affect the amount/ percentage of detected genomic alterations in circualting cell-free DNA in blood include tumor growth, turn-over, size, heterogeneity, vascularization, disease progression, or treatment

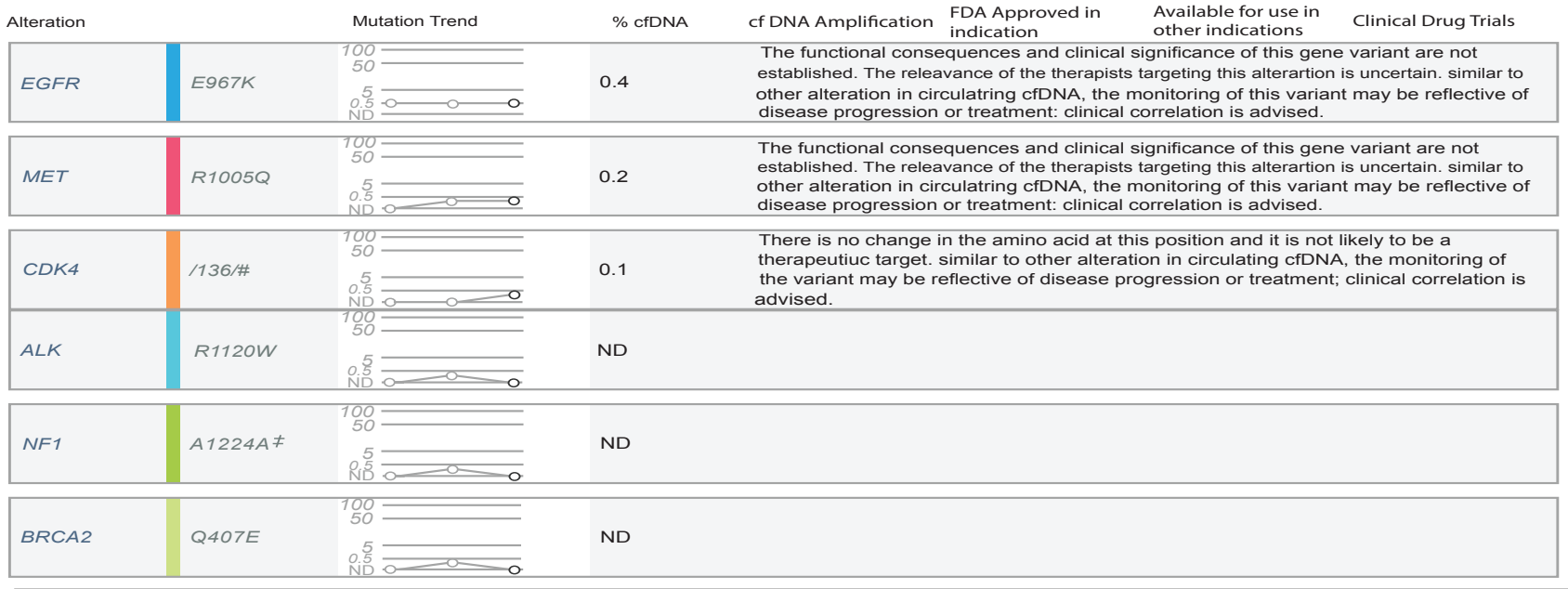

GUARDANT HEALTH | Arthur Baca, MD phd Labaratory Director CLIA ID: 05D2070300 505 Penobscot Drive, Redwood city, CA 94063 T:855-698-8887 clientservices@ @ guardanthealth.com https://portal.guardanthealth.com TST-PRT-001 V13.0 PG 1 OF 6

Figure 1: Report showing the case study of case 1 
Citation: Nezami MA, Hager S, Garner J (2016) The Role of Telomeres in Cancer Development and Progression, and the Double Edge Sword Effect with Tamoxifen. Biol Med (Aligarh) 8: 296. doi: 10.4172/0974-8369.1000296

\section{Breast cancer with positive NF-1}

43 year old female with history of breast ductal carcinoma, 100 percent ER and PR positive, and a Her-2 negative. Her Ki67 was 80 percent; SBR on biopsy was determined to be $8 / 9$.

She had exhausted many alternative cancer therapies since $5 / 2012$, when she was diagnosed first. None of the therapies was effective, and in fact her cancer grew to $4 \mathrm{~cm}$, and she opted for bilateral mastectomy and axial lymph node resection in November 2012, which was positive for $2 \mathrm{LN}$ invasions. Upon her arrival at our clinic, she had recurrence of her tumor, with two large palpable sites in the chest on skin. She was complaining of a new onset consistent and dry cough, and was concerning for lung metastasis. Upon evaluation, we ordered a PET scan, which showed multiple pulmonary metastasis bilaterally, which were too numerous to count, with an SUV max of 4.8. She had multiple masses in right breast with max SUV of 12.9, and multiple smaller lesions with max SUV of 5.5, plus mediastinal and internal mammary

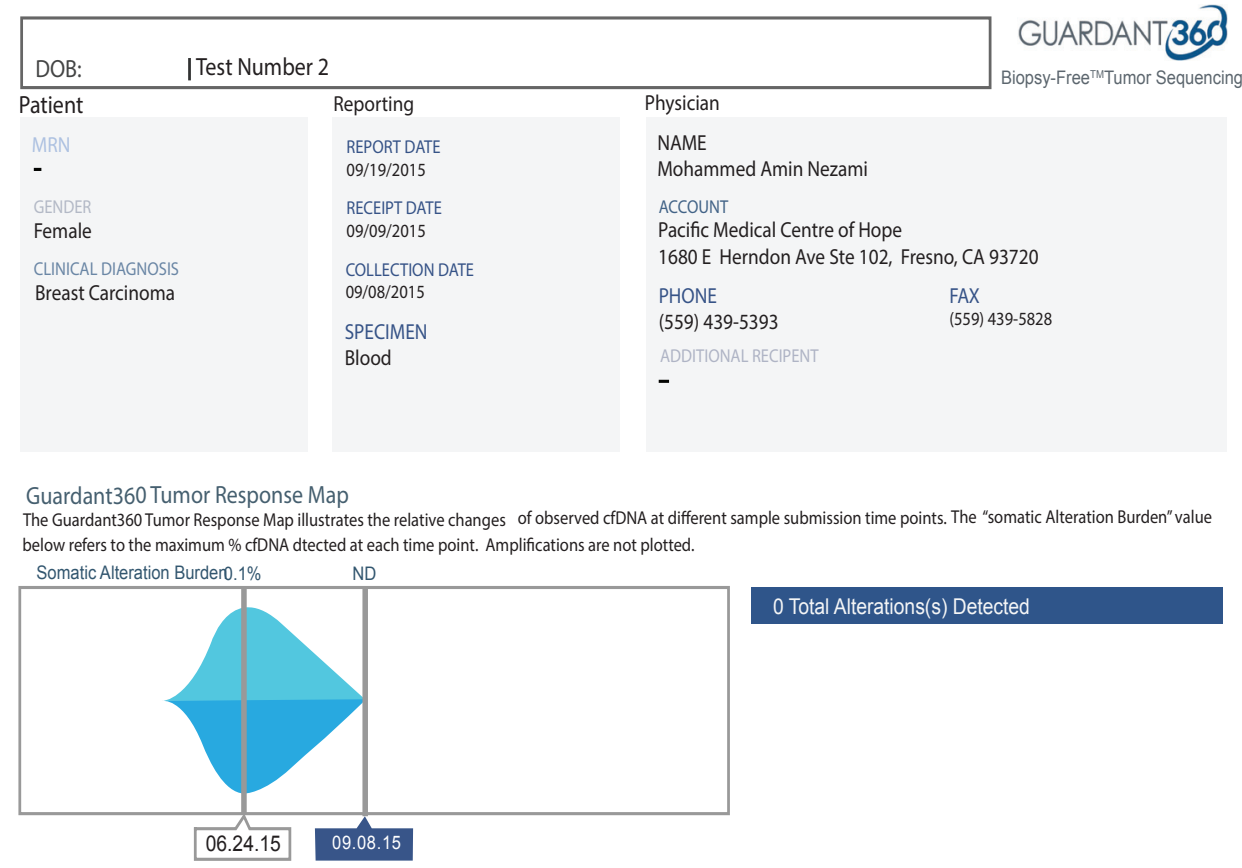

Genomic Alterations: Not Detected (ND). Genomic alterations may be present that are below the limit of detection of this test. Certain sample or variant characterstics may result in reduced anaytic sensitivity, such as poor sample quality or improper collection. Genomic alterations in a tumor may be present, but are not detected in circulating cellfrre DNA from this blood specimen with this test

Summary of Alterations \& Associated Treatment Options

The percenatage, or allele frequency, of altered cell -free DNA (\% of DNA) circulating in blood is related to unique tumor biology of this patient. Factors that may effect the amount/ percentages of detected genomic alterations in circulating cell-free DNA in blood include tumor growth, turn -over, size, heterogeneity, vascularization, disease progression, or treatment.

\begin{tabular}{|c|c|c|c|c|c|c|c|}
\hline Alteration & & Mutation Trend & $\%$ cfDNA & cf DNA Amplification & $\begin{array}{l}\text { FDA Approved in } \\
\text { indication }\end{array}$ & $\begin{array}{l}\text { Available for use in } \\
\text { other indications }\end{array}$ & clinical Drug Trials \\
\hline NF1 & $R 1362 Q$ & $\begin{array}{l}100 \overline{ } \\
50 \\
0.5 \bar{\square} \\
\square\end{array}$ & ND & & & & \\
\hline$A P C$ & $S 2632 L$ & $\begin{array}{l}100= \\
50= \\
5 \overline{=} \\
0.5=\square\end{array}$ & ND & & & & \\
\hline
\end{tabular}

For a more detailec Guardant360 patient Report, log onto: http://portal.guardanthealth.com

To set up an account, contact Cl ent Services: 855.698 .8887

The chart above annotates the percentage or allele frequency, of altered circulating cell free-DNA (\% cfDNA) detected in this patient. The detected genomic alterations are listed in descending order by $\%$ by gene.

The "FDA Approved in indication "and "Available for use in other indications" columns describe drugs associated with specific fenomic alterations. It based on publicly available information as described in the " Detailed Therapy Results" and "clincial Relevance of Detected Alterations" sections of the report

Definitions

None.

\begin{tabular}{l|l} 
GUARDANT HEALTH & Arthur Baca, MD phd Laboratory Director/2686 Middlefield Rd, Sube C, D, E. Redwood city, CA 94063 \\
T: 855-698-887 clientservices@guardanthealth.com https://portal.guardanthealth.com Report Version 4.1 TST-PRT-001 V12.0 pg 1 of 4
\end{tabular}

Figure 2: Report showing the case study of case 2 
Citation: Nezami MA, Hager S, Garner J (2016) The Role of Telomeres in Cancer Development and Progression, and the Double Edge Sword Effect with Tamoxifen. Biol Med (Aligarh) 8: 296. doi: 10.4172/0974-8369.1000296

LNs in chest and supraclavicular LNs. In additional she had multiple metastatic LNs in right axilla with SUV max of 2.9.

She had tumor markers all elevated in labs. Immediately she was started on IV epigenetic therapies which she received on daily basis. After 10 treatments, her labs were rechecked. The results showed significant decreases in all her tumor markers. Her cough improved, and she maintained her treatments at our clinic. She did not change her diet, nor did she start any chemotherapy. At this time, she was not on any hormonal blockade, confirming the independent response to epigenetic therapy. In November 2014, she had a restaging PET scan, showing marked reduction of the activities in mediastinal and internal mammary LNs, with improved diffuse pulmonary metastasis. The PET scan was performed and compared with PRE treatment scan after 6 weeks of therapy. The following PET scan done on $1 / 12 / 15$, demonstrated complete resolution of all pulmonary metastasis and axillary/supraclavicular lymph nodes. There was minimal residual disease reported in the mediastinal lymph nodes, with SUV activity decreased to 5 from 10.6, and to 3 from 7 . At this time, the tumor markers were substantially reduced and most returned to normal limits. CA 15.3 was at 31 , previously 37,52 , and 56 (respective dates: 4/7/15, $1 / 16 / 15,11 / 21 / 14,10 / 19 / 14)$. CA 27.29 was 33 , previously $44,47,55.6$, and 65 (respective dates $11 / 21 / 14,10 / 9 / 14,9 / 11 / 14,8 / 8 / 14$ ). CA 125 was 18.6, previously 19.5 and 39.8 (respective dates 4/7/15, 1/16/15 and 10/9/14). At this time she was receiving combination epigenetic therapies with hormonal blockade, consisting of GnRH blockade (Lupron) as well as Tamoxifen at $5 \mathrm{mg}$ every other day (1/8 normal dose of $20 \mathrm{mg}$ per day). She was restaged again with a PET/CT on 4/15/2015, which showed progression of the local disease in surgical incision, but only minimal residual disease and faint FDG activity (SUV activity of 1.5 compared to 4.8 pre-treatment) in her mediastinal pulmonary lesions, with complete resolution of liver findings. Due to the fact that she was down staged from a Stage IV disease to a local disease in the breast, it was justified to remove the breast tissue and treat her locally with surgery.

Following her surgery, her circulatory tumor DNA was evaluated through Guardant 360, which revealed positive EGFR. Subsequently the patient was started on Tarceva, and the Guardant 360 test was repeated. Although the EGFR mutation allele fraction (MAF) decreased with therapy, the second test showed NF-1, ALK, EGFR and cMET (Figure 2). The presence of NF-1 confirmed the mutated oncosuppressor gene, likely due to the exposure to anti-estrogen therapy, an example of induced heterogeneity. The presence of other alterations, confirmed the induction of heterogeneity by Tarceva. The presence of NF-1 and other alterations, although considered poor prognostic markers, did not change this patient's outcome, or her disease free survival. We believe that this success was due to the combination of epigenetic therapies.

This case suggests an independent, as well as synergistic method of epigenetic therapies combined with hormonal blockade, which was able to treat non-operable Stage IV breast cancer, and down stage the patient successfully to treat the local disease. This quick response suggests further need to evaluate this novel combinational approach in treating resistant and recurrent advanced breast cancer.

\section{Conclusion}

Tamoxifen has been proven to prevent invasive and noninvasive breast cancer $[1,2]$. Retrospective studies also indicate an effect on incidence of endometrial and ovarian cancer [11,12]. We also recommend large scale studies using Tamoxifen for prevention of cancer. Perhaps the most important question that remains to be answered is the timing of Tamoxifen administration in the prevention of cancer.

\section{References}

1. Powles TJ, Ashley S, Tidy A, Smith IE, Dowsett M (2007) Twenty-Year Followup of the Royal Marsden Randomized, Double-Blinded Tamoxifen Breast Cancer Prevention Trial. J Natl Cancer Inst 99: 283-290.

2. Cuzick J, Forbes JF, Sestak I, Cawthorn S, Hamed H, et al. (2007) Long-term results of tamoxifen prophylaxis for breast cancer--96-month follow-up of the randomized IBIS-I trial. J Natl Cancer Inst 99: 272-282.

3. Blackburn EH (2005) Telomerase and Cancer: Kirk A. Landon--AACR prize for basic cancer research lecture. Mol Cancer Res 3: 477-482.

4. Belair CD, Yeager TR, Lopez PM, Reznikoff CA (1997) Telomerase activity: a biomarker of cell proliferation, not malignant transformation. Proc Natl Acad Sci USA 94: 13677-13682

5. Lu L, Zhang C, Zhu G, Irwin M, Risch H, et al. (2011) Telomerase expression and telomere length in breast cancer and their associations with adjuvant treatment and disease outcome. Breast Cancer Res 13: R56.

6. Wang Z, Kyo S, Maida Y, Takakura M, Tanaka M, et al. (2002) Tamoxifen regulates human telomerase reverse transcriptase (hTERT) gene expression differently in breast and endometrial cancer cells. Oncogene 21: 3517-3524.

7. Henson JD, Neumann AA, Yeager TR, Reddel RR (2002) Alternative lengthening of telomeres in mammalian cells. Oncogene 21: 598-610.

8. Blocking Telomerase Kills Cancer Cells but Provokes Resistance, Progression (2012) Drug Week

9. Xu T, Lu B, Tai Y, Goldkorn A (2010) A Cancer Detection Platform Which Measures Telomerase Activity from Live Circulating Tumor Cells Captured on a Microfilter. Cancer Research 70: 6420-6426.

10. Fizazi K, Morat L, Chauveinc L, Prapotnich D, De Crevoisier R, et al. (2007) High detection rate of circulating tumor cells in blood of patients with prostate cancer using telomerase activity. Ann Oncol 18: 518-521.

11. Vrscaj MU, Kovacic J, Bebar S, Djurisic A, Fras P, et al. (2001) Endometrial and other primary cancers after tamoxifen treatment of breast cancer — results of retrospective cohort study. Eur J Obstet Gynecol Reprod Biol 95: 105-110.

12. Fisher B, Costantino JP, Wickerham DL, Redmond CK, Kavanah M, et al. (1998) Tamoxifen for Prevention of Breast Cancer: Report of the National Surgical Adjuvant Breast and Bowel Project P-1 Study. J Natl Cancer Inst 90: 1371-1388. 